\title{
Outliers: The Case for Individualized Therapy
}

\author{
Richard L Slaughter* \\ College of Pharmacy and Health Sciences, Wayne State University, Detroit, MI 48201, USA
}

An outlier is a scientific term to describe things or phenomena that lie outside normal experience. In pharmacokinetics this would be a parameter such as drug clearance or half-life that has a value that is much different from the average. Patients with pharmacokinetic parameters that are outliers are often the most difficult to manage. Identifying the reasons for being an outlier has always been a goal in the field of pharmacokinetics/pharmacodynamics. One of the challenges of modern medicine is to accurately identify the cause of outliers, thus allowing that for better targeted drug selection and dosage. This is the basic principles of what today is called individualized medicine. The clinical relevance of variability depends on the specific drug or drug class in question. As an example, some drug classes have wide therapeutic ranges and response is easily monitored and as such do not need an individualized approach to therapy. Examples include antihypertensive such as the angiotensin-enzyme converting inhibitors or angiotensin receptor blockers. Similarly certain antimicrobial agents are much simpler to use than others. The fluoroquinolones are easier and generally safer to use than aminoglycosides and vancomycin with the latter 2 classes of antibiotics requiring a individualized approach to manage therapy. It is fortunate that the number of drugs that actually require individualized attention is relatively small. The 2011 edition of Pharmacotherapy: A Pathophysiologic approach lists 18 selected drugs that are monitored routinely with blood concentrations of which only 4 drugs (carbamazepine, Phenobarbital, phenytoin and digoxin) in the in the top 200 drugs in 2012 presented by RxList [1,2]. This editorial will argue that despite a very large volume of literature and much improved understanding of the causes of pharmacokinetic variability that there is still much to learn about those factors that are important in determining initial drug dosage selection. Specifically, identifying those factors that are responsible for extremes seen in variance or the causes of being an outlier. This will be illustrating using 2 old standards that have been studied for at least the past 4 decades, vancomycin and warfarin.

Vancomycin is the mainstay of therapy used for the treatment of gram positive infections, specifically those caused by methicillinresistant Staphylococcal aureus. Its effective use is limited because of nephrotoxicity and ototoxicity which can be effectively managed through appropriate monitoring and dosing strategies. The pharmacokinetics of vancomycin has been studied for over 30 years with more manuscripts published each year now than 20 years ago. A recent PubMed search showed over 1400 publications under the terms "vancomycin pharmacokinetics" and over 500 publications under the terms "vancomycin dosing". In years 2007-2011, 76 articles per year were published under the terms "vancomycin pharmacokinetics" compared to 34 per year in the years 1987-1991. Clearly issues concerning the evaluation of the pharmacokinetics of vancomycin are not yet fully understood and remain more relevant today than 20 years ago. In fact there is a recent review published in 2011 titled "Vancomycin a 50-something-year-old antibiotic that we do not fully understand" [3]. In 2009 a consensus guideline was developed to assist clinicians with dosing and monitoring vancomycin therapy [4]. This guideline grew out of a understanding that proper vancomycin administration requires more aggressive dosing with more intensive monitoring in order to achieve optimal outcomes. This guideline recommends targeting therapy to an AUC (area under the concentration time curve): MIC
(Minimum inhibitory concentration) ratio of 400 and those monitoring trough vancomycin concentrations are the most practical method to use to monitor vancomycin therapy. Trough concentrations maintained between 15 and $20 \mathrm{mcg} / \mathrm{ml}$ with $S$. aureus infections that have an MIC below $2 \mathrm{mcg} / \mathrm{ml}$ should achieve this goal. Alternative therapies are recommended when $S$. aureus infections have a vancomycin MIC above $2 \mathrm{mcg} / \mathrm{ml}$. This guideline concludes that more clinical experience is yet needed to determine the impact that these guidelines have on vancomycin toxicity.

A recent review of population pharmacokinetic analyses of vancomycin has identified the major sources of variance in its pharmacokinetics [5]. In adult patients, vancomycin clearance depends on weight and creatinine clearance while in pediatric patients age is an additional parameter to be considered. Through inclusion of these covariates the mean value of the interindividual variability in vancomycin clearance was 30\% (range: 15.6-45\%) and the mean (range) value of the residual variability was $20 \%$ (7-39.6\%). Use of age, weight and creatinine clearance in dosage selection will improve accuracy of dosage selection but with the degree of accuracy shown, will still require follow up serum concentration monitoring to achieve optimum response. Another recent study [6] in patients with congestive heart failure showed, using multiple regression analysis, that vancomycin clearance was related to $\left(r^{2}=0.649\right)$ LVEF (left ventricular ejection fraction), creatinine clearance and body weight. This still left $35 \%$ of the variance in vancomycin clearance not explained. Unexplained variability in clearance is one reason studies continue to show that patients receiving vancomcyin are exposed to high trough concentrations (a risk factor for nephrotoxicity). For example, a recent prospective multicenter trial showed that almost $30 \%$ of patients dosed by standard care had trough values above $15 \mathrm{mcg} / \mathrm{ml}$, a risk factor for developing vancomycin induced nephrotoxicity [7]. Identifying additional factors beyond creatinine clearance, weight and that explain why patients have clearance values that outliers continue to be a challenge with vancomycin. This remains one of the reasons for the continued need for patients to be monitored by skilled professionals in pharmacokinetics so that patients receiving vancomycin are appropriately managed.

Large variations in pharmacokinetics can be explained through genetic variation in drug metabolizing enzymes and transporter proteins. An example includes drug hypersensitivity in patients with

*Corresponding author: Richard L Slaughter, M.S., FCCP, Associate Dean Pharmacy, Eugene Applebaum College of Pharmacy and Health Sciences, Wayne State University, Detroit, Ml 48201, USA, Tel: 313 577-8502; Fax: 313 577-9457; E-mail: rls@wayne.edu

Received September 21, 2012; Accepted September 22, 2012; Published September 24, 2012

Citation: Slaughter RL (2012) Outliers: The Case for Individualized Therapy. Adv Pharmacoepidem Drug Safety S1:e001. doi:10.4172/2167-1052.S1-e001

Copyright: ( 2012 Slaughter RL. This is an open-access article distributed under the terms of the Creative Commons Attribution License, which permits unrestricted use, distribution, and reproduction in any medium, provided the original author and source are credited. 
gene duplication of the CYP2D6 enzyme (ultrafast metabolizers (UM)). In an extreme case, the death of an infant from morphine toxicity resulted from being breast fed by a mother with the UM CY2D6 phenotype who received relatively modest doses of codeine (ie, $60 \mathrm{mg}$ twice a day) [8]. Poor analgesic response to codeine appears to be related to the presence of CYP2D6 alleles associated with poor metabolizers (PMs) (i.e., the CYP2D6 ${ }^{\star} 3,{ }^{\star} 4,{ }^{\star} 5,{ }^{\star} 6$ and ${ }^{\star} 7$ alleles) $[9,10]$. Tamoxifen patients who have the CYP2D6 alleles ${ }^{\star} 4,{ }^{\star} 5,{ }^{*} 10$ and ${ }^{*} 41$ (which are associated with decreased formation of antiestrogen metabolites) have a poorer response to tamoxifen in treating breast cancer [11]. Another example, is the relationship between reduced effectiveness to clopridogrel treatment in patients who are poor metabolizers with the inherited CYP2C19 ${ }^{*} 2$ and CYP2C19 $* 3$ alleles [12]. The FDA package insert recommends that clopridogrel therapy be avoided in patients with impaired CYP2C19 function [13].

The importance of illustrating how genetics can assist in the identification of an outlier can be well exemplified by warfarin. This is a drug that has been a mainstay of anticoagulation therapy for decades. In PubMed under the search term "warfarin pharmacokinetics" there is 1385 articles cited over a 4 decade period. Complexities associated with appropriate dosing and management is well founded and yet after 4 decades is not yet completely understood. Pharmacogenomic studies in recent years have, however, provided good insight into reasons why certain patients are outliers in response. Genotypic variation in the CYP2C9 (metabolizes warfarin) and VKORC1 (vitamin K epoxide reductase complex subunit 1 ; the target for warfarin inactivation of vitamin K) accounts for $30-40 \%$ of the variability in response to warfarin. Patients with the CYP $2 \mathrm{C} 9{ }^{*} 2$ or $* 3$ variant alleles require lower doses and as do patients with certain single nucleotide polymorphisms in the VKORC1 gene (ie the $-1639 \mathrm{G}>\mathrm{A}$ allele) [14]. Pharmacogenomic based dosing correctly predicts patients requiring extreme doses of warfarin almost 2 fold better than a clinical algorithm [15]. The IWPC pharmacogenomic algorithm which can be downloaded at www. pharmgkb.org [16] accounts for age, weight, height, CYP2C9 genotype, VKORC1 genotype, ethnicity and the presence of drug interactions(i.e., inducers and amiodarone). Three methods of pharmacogenomic dosing of warfarin were compared and it was found that dosing accuracy is improved with pharmacogenomic dosing. However they concluded that the degree of accuracy still did not support routine utilization of genotypic dosing [17]. This is illustrated in a recent study that showed suboptimum performance of the International Warfarin Pharmacogenomic Consortium model15 in Israeli patients. Only $27 \%$ of the variance in dose was accounted for based on this model. Incorporation of the VKORC1 D36Y variant, which is not accounted for in the IWPC model, improved model performance by explaining $47.2 \%$ of the variance in dose. Identifying the cause of a patient's warfarin dose being an outlier still remains a large therapeutic challenge. This is exemplified by very large dose ranges seen by VKORC1 genotype. For the VKORC1-1639 AA genotype the weekly dose may be as low as $5 \mathrm{mg} /$ week or as high as $50 \mathrm{mg} /$ week, while for the VKORC1-1639 GG genotype the weekly dose ranges from $10 \mathrm{mg} /$ week to $100 \mathrm{mg} /$ week.

These examples indicate how initial dose selection and our understanding of desired endpoints have improved over many years. Despite this we still do not fully understand all the variables associated in patients who are outliers and are at the highest risk for therapeutic failure or toxicity. It will always be a goal to identify characteristics of patients that are outliers. These examples also illustrate that despite a large volume of literature that help to improve initial dosage selection that active strategies via therapeutic monitoring of desired endpoints is still required. Therapeutic drug monitoring remains a necessity. At least for these 2 drugs the only way to insure efficacy and minimize toxicity is through an individualized approach to therapy guided by skilled health care professionals who have expertise in pharmacokinetics and pharmacogenomics.

\section{References}

1. Bauer LA (2011) Clinical Pharmacokinetics and Pharmacodynamics Pharmacotherapy: A Pathophysiologic Approach. 8th ed. New York: McGrawHill Chapter 8.

2. http://www.rxlist.com/script/main/art.asp?articlekey $=79510$

3. Schilling A, Neuner E, Rehm SJ (2011) Vancomycin: A 50-something-year-old antibiotic we still don't understand. Cleve Clin J Med 78: 465-471.

4. Rybak MJ, Lomaestro BM, Rotschafer JC, Moellering RC, Craig WA, et al (2009) Therapeutic monitoring of vancomycin in adults Summary of consensus recommendations from the American Society of Health-System Pharmacists, the Infectious Diseases Society of America, and the Society of Infectious Diseases Pharmacists. Pharmacotherapy 29: 1275-1279.

5. Marsot A, Boulamery A, Bruguerolle B, Simon N (2012) Vancomycin: a review of population pharmacokinetic analyses. Clin Pharmacokinet 51: 1-13.

6. Shimato Y, Fukuda T, Tominari S, Fukumoto K, Ueno K, et al. (2012) Decreased vancomycin clearance in patients with congestive heart failure. Eur J Clin Pharmacol.

7. Bosso JA, Nappi J, Rudisill C, Wellein M, Bookstaver PB, et al. (2011) Relationship between vancomycin trough concentrations and nephrotoxicity: a prospective multicenter trial. Antimicrob Agents Chemother 55: 5475-5479.

8. Madadi P, Koren G, Cairns J, Chitayat D, Gaedigk A, et al. (2007) Safety of codeine during breastfeeding. Can Fam Physician 53: 33-35.

9. MA JD, Lee KC, Kuo GM (2012) Clinical application of pharmacogenomics. J Pharmacy Practice 25: 417-427.

10. VanderVaart S, Berger H, Sistonen J, Madadi P, Matok I, et al. (2011) CYP2D6 polymorphisms and codeine analgesia in postpartum pain management: a pilot study. Ther Drug Monit 33: 425-432.

11. Schroth W, Antoniadou L, Fritz P, Schawb M, Muerdter T, et al. (2007) Breast cancer treatment outcome with adjuvant tamoxifen relative to patient CYP2D6 and CYP2C19 genotypes. J Clin Oncol 25: 5187-5193.

12. Malek LA, Przyluski J, Spiewak M, Kłopotowski M, Kostrzewa G, et al. (2010) Cytochrome P450 2C19 polymorphism, suboptimal reperfusion and all-cause mortality in patients with acute myocardial infarction. Cardiology 117: 81-87.

13. http://www.accessdata.fda.gov/drugsatfda_docs/label/2009/020839s044lbl. pdf, accessed September 19, 2012.

14. Wang L, McLeod HL, Weinshilboum RM (2011) Genomics and drug response. N Engl J Med 364:1144-1153.

15. International Warfarin Pharmacogenetics Consortium, Klein TE, Altman RB Eriksson N, Gage BF, Kimmel SE, et al. (2009) Estimation of the warfarin dose with clinical and pharmacogenetic data. N Engl J Med 360:753-764.

16. www.pharmgkb.org/drug/PA541906, accessed September 20, 2012.

17. Shaw PB, Donovan JL, Tran MT, Lemon SC, Burgwinkle P, et al. (2010) Accuracy assessment of pharmacogenetically predictive warfarin dosing algorithms in patients of an academic medical center anticoagulation clinic. J Thromb Thrombolysis 30: 220-225. 